\title{
SHARP LOGARITHMIC DERIVATIVE ESTIMATES WITH APPLICATIONS TO ORDINARY DIFFERENTIAL EQUATIONS IN THE UNIT DISC
}

\author{
I. CHYZHYKOV, J. HEITTOKANGAS ${ }^{\bowtie}$ and J. RÄTTYÄ
}

(Received 25 June 2009; accepted 4 January 2010)

\author{
Communicated by P. C. Fenton
}

\begin{abstract}
New estimates are obtained for the maximum modulus of the generalized logarithmic derivatives $f^{(k)} / f^{(j)}$, where $f$ is analytic and of finite order of growth in the unit disc, and $k$ and $j$ are integers satisfying $k>j \geq 0$. These estimates are stated in terms of a fixed (Lindelöf) proximate order of $f$ and are valid outside a possible exceptional set of arbitrarily small upper density. The results obtained are then used to study the growth of solutions of linear differential equations in the unit disc. Examples are given to show that all of the results are sharp.
\end{abstract}

2000 Mathematics subject classification: primary 34M10; secondary 30D30.

Keywords and phrases: logarithmic derivative, proximate order, order of growth, linear differential equation, growth of solutions.

\section{Introduction and statement of results}

Let $\mathbb{D}=\{z:|z|<1\}$ be the open unit disc in the complex plane $\mathbb{C}$. The space of all analytic functions in $\mathbb{D}$ is denoted by $A(\mathbb{D})$. The order of growth of a function $f \in A(\mathbb{D})$ is defined as

$$
\sigma_{M}(f)=\limsup _{r \rightarrow 1^{-}} \frac{\log ^{+} \log ^{+} M(r, f)}{|\log (1-r)|},
$$

where $M(r, f)=\max _{|z|=r}|f(z)|$ is the maximum modulus of $f$.

Definition 1.1. Let $f \in A(\mathbb{D})$ be such that $\sigma_{M}(f) \in(0, \infty)$. Then a continuous function $\sigma:[0,1) \rightarrow[0, \infty)$ is a (Lindelöf) proximate order of $f$, provided that it satisfies the following conditions.

This research was supported in part by the Academy of Finland \#210245 and \#121281; the Väisälä Fund of the Finnish Academy of Science and Letters; MEC-Spain MTM2005-07347, MTM2006-26627-E and MTM2007-30904-E; and the European Science Foundation RNP HCAA.

(C) 2010 Australian Mathematical Publishing Association Inc. 1446-7887/2010 \$16.00 
(A) There exists $R_{0}>0$ such that $\sigma$ is positive and differentiable in $\left[R_{0}, 1\right)$.

(B) $\lim _{r \rightarrow 1^{-}} \sigma(r)=\sigma_{M}(f)$.

(C) $\lim _{r \rightarrow 1^{-}} \sigma^{\prime}(r)(1-r)|\log (1-r)|=0$.

(D) $\lim \sup _{r \rightarrow 1^{-}}(1-r)^{\sigma(r)} \log M(r, f)=1$.

It is known that every function $f \in A(\mathbb{D})$ with $\sigma_{M}(f) \in(0, \infty)$ has a proximate order, and that proximate orders are not unique [12, 17].

Sharp growth estimates for the maximum modulus of the generalized logarithmic derivative $f^{(k)} / f^{(j)}$, where $f$ is meromorphic in $\mathbb{D}$ and $k$ and $j$ are integers satisfying $k>j \geq 0$, are obtained in [3,8]. The special cases where $f$ either belongs to the Nevanlinna class or is a Blaschke product are further discussed in [9]. In addition, growth estimates for integral means of generalized logarithmic derivatives of meromorphic functions are obtained in $[4,10]$. This paper deals with functions $f \in A(\mathbb{D})$ of finite order of growth, and the corresponding estimates for the generalized logarithmic derivatives are stated in terms of a fixed proximate order of $f$. To the best of our knowledge these estimates are the first sharp estimates in terms of maximum modulus.

The main result is stated as follows.

TheOREM 1.2. Let $f \in A(\mathbb{D})$ be such that $\sigma_{M}(f)<\infty$. If $\sigma_{M}(f)>0$, let $\sigma$ be a proximate order of $f$. Let $k$ and $j$ be integers satisfying $k>j \geq 0$, and let $\delta$, $\varepsilon \in(0,1)$. Then there exists a countable collection of discs $D_{v}=\left\{w:\left|w-z_{v}\right|<r_{v}\right\}$, where $r_{v}<1-\left|z_{v}\right|$, and a constant $C>0$ such that

$$
\sum_{R<\left|z_{v}\right|<1} r_{\nu} \leq \delta(1-R) \quad \text { as } R \rightarrow 1^{-},
$$

and

$$
\left|\frac{f^{(k)}(z)}{f^{(j)}(z)}\right| \leq \begin{cases}C\left(\frac{|\log (1-|z|)|}{\delta(1-|z|)^{\sigma(|z|)+1}}\right)^{k-j} & \text { if } \sigma_{M}(f)>1, \\ \left(\frac{1}{\delta(1-|z|)}\right)^{2(k-j)+\varepsilon} & \text { if } \sigma_{M}(f) \leq 1,\end{cases}
$$

for all $z \in \mathbb{D} \backslash \bigcup_{v} D_{v}$.

The rotated projection on $[0,1)$ of the exceptional set in Theorem 1.2 is of arbitrarily small upper density. For a measurable set $E \subset[0,1)$, the upper density is defined as

$$
\bar{D}(E)=\limsup _{r \rightarrow 1^{-}} \frac{m(E \cap[r, 1))}{1-r},
$$

where $m(F)$ is the Lebesgue measure of the set $F$.

COROLLARY 1.3. Under the assumptions of Theorem 1.2 there exists an exceptional set $E \subset[0,1)$ with $\bar{D}(E) \leq 2 \delta$ such that

$$
\left|\frac{f^{(k)}(z)}{f^{(j)}(z)}\right| \leq\left(\frac{1}{1-|z|}\right)^{\left(\max \left\{\sigma_{M}(f), 1\right\}+1\right)(k-j)+\varepsilon}
$$

for all $z \in \mathbb{D}$ satisfying $|z| \notin E$. 
As a nontrivial application of Corollary 1.3, we study the growth of the solutions of the linear differential equation

$$
f^{(k)}(z)+A_{k-1}(z) f^{(k-1)}(z)+\cdots+A_{1}(z) f^{\prime}(z)+A_{0}(z) f(z)=0,
$$

where the coefficients $A_{0}, \ldots, A_{k-1}$ belong to $A(\mathbb{D})$. The growth of the solutions of (1.5) in the case of entire coefficients is typically dealt with using Wiman-Valiron theory; see [15] and the references therein. The classical Wiman-Valiron analogue in the unit disc as presented in [12] seems to be an insufficient tool for dealing with the solutions of (1.5). However, recent developments in Wiman-Valiron theory have been successfully applied to the theory of differential equations in the unit disc [5, 6].

For $0 \leq q<\infty$, the weighted Hardy space $H_{q}^{\infty}$ consists of those functions $A \in$ $A(\mathbb{D})$ for which

$$
\sup _{0 \leq r<1} M(r, A)\left(1-r^{2}\right)^{q}<\infty .
$$

We note that the differential equation (1.5) with coefficients in weighted Hardy spaces were studied in $[11,18]$. Moreover, $A$ is said to belong to $G_{p}$ if

$$
p=\inf \left\{q \geq 0: A \in H_{q}^{\infty}\right\}
$$

see [3]. It is clear that

$$
G_{p}=\bigcap_{q>p} H_{q}^{\infty} \backslash \bigcup_{q<p} H_{q}^{\infty},
$$

and further, $A \in G_{p}$ if and only if

$$
p=\limsup _{r \rightarrow 1^{-}} \frac{\log ^{+} M(r, A)}{|\log (1-r)|} .
$$

The findings concerning (1.5) are summarized in the following theorem, which partially improves the corresponding results in [2, 3, 13]. Note that (1.7) below is proved in [13], but it has been included here for the sake of completeness.

THEOREM 1.4. Let $f$ be a solution of (1.5), where $A_{j} \in G_{p_{j}}$ for $j=0, \ldots, k-1$. Let $1 \leq \alpha<\infty$, and denote $p_{k}=0$.

(a) Then all solutions $f$ of (1.5) satisfy

$$
\sigma_{M}(f) \leq \max \left\{0, \max _{0 \leq j \leq k-1}\left\{\frac{p_{j}}{k-j}-1\right\}\right\}
$$

and

$$
\max \left\{\sigma_{M}(f), 1\right\} \geq \min _{1 \leq j \leq k}\left\{\frac{p_{0}-p_{j}}{j}-1\right\}
$$

(b) Suppose that

$$
\min _{1 \leq j \leq k}\left\{\frac{p_{0}-p_{j}}{j}\right\} \geq 2
$$


Then $\sigma_{M}(f) \leq \alpha$ if and only if

$$
\max _{0 \leq j \leq k-1}\left\{\frac{p_{j}}{k-j}-1\right\} \leq \alpha .
$$

(c) Suppose that (1.9) holds. If $n \in\{0, \ldots, k-1\}$ is the smallest index for which

$$
\frac{p_{n}}{k-n}=\max _{0 \leq j \leq k-1}\left\{\frac{p_{j}}{k-j}\right\},
$$

then in every solution basis of (1.5) there are at least $k-n$ linearly independent solutions $f$ such that

$$
\sigma_{M}(f)=\max _{0 \leq j \leq k-1}\left\{\frac{p_{j}}{k-j}-1\right\} .
$$

In addition to $\sigma_{M}(f)$ defined in (1.1), the quantity

$$
\sigma_{m}(f)=\limsup _{r \rightarrow \infty} \frac{\log ^{+} m(r, f)}{|\log (1-r)|},
$$

where $m(r, f)$ is the Nevanlinna proximity function of $f$, is often used to describe the growth of an analytic function in the unit disc. Although in the complex plane the analogously defined growth orders are equal for all entire functions, in the unit disc we only have the inequalities

$$
\sigma_{m}(f) \leq \sigma_{M}(f) \leq \sigma_{m}(f)+1 .
$$

The inequalities in (1.13) are known to be best possible in the sense that there exist $g$, $h \in A(\mathbb{D})$ such that $\sigma_{m}(g)=\sigma_{M}(g)$ and $\sigma_{M}(h)=\sigma_{m}(h)+1$.

Theorems 1 and 2 in [14] together form an analogue of Theorem 1.4 above stated in terms of the growth order $\sigma_{m}(f)$. Despite the close connection, the results in [14] do not imply Theorem 1.4 above, and vice versa. The growth of logarithmic derivatives appearing in the proof of [14, Theorem 2] is dealt with [10, Lemma 3.1]. Since this lemma compares the logarithmic derivative to $\log ^{+} m(r, f)$ rather than to $\log ^{+} M(r, f)$, it is not useful in proving Theorem 1.4. We also point out that Theorems 1 and 2 in [14] were generalized in [4].

Corollary 1.5. Let $A_{j} \in G_{p_{j}}$ for $j=0, \ldots, k-1$. If

$$
\max _{0 \leq j \leq k-1}\left\{\frac{p_{j}}{k-j}-1\right\}=\frac{p_{0}}{k}-1 \geq 1,
$$

then all nontrivial solutions $f$ of (1.5) satisfy $\sigma_{M}(f)=p_{0} / k-1$.

The assumption (1.14) and the estimate (4.3) below show that (1.9) and (1.11), for $n=0$, are satisfied. Thus all functions $f_{j}$ in every solution basis $\left\{f_{1}, \ldots, f_{k}\right\}$ 
of (1.5) satisfy $\sigma_{M}\left(f_{j}\right)=p_{0} / k-1$. If $f$ is a nontrivial solution of (1.5), then there exist constants $C_{1}, \ldots, C_{k} \in \mathbb{C}$ such that $f=C_{1} f_{1}+\cdots+C_{k} f_{k}$ and that $C_{j_{0}} \neq 0$ for some $j_{0} \in\{1, \ldots, k\}$. Therefore $f$ and the functions $f_{j}, j \neq j_{0}$, form another solution basis of (1.5), and hence $\sigma_{M}(f)=p_{0} / k-1$. This proves Corollary 1.5.

The remainder of this paper is organized as follows. Section 2 is devoted to examples which prove the sharpness of Theorems 1.2 and 1.4. Sections 3 and 4 contain the proofs of Theorems 1.2 and 1.4, respectively.

\section{Sharpness discussion}

The sharpness of Theorem 1.2 is illustrated by the following example.

EXAMPLE 1. (a) For $1 \leq \alpha<\infty$, the function

$$
f(z)=\exp \left(\frac{1}{(1-z)^{\alpha}} \log \frac{1}{1-z}\right)
$$

satisfies

$$
\log M(r, f)=(1-r)^{-\alpha}|\log (1-r)|
$$

for all $0<r<1$, and therefore $\sigma_{M}(f)=\alpha$. The function

$$
\sigma(r)=\alpha+\frac{\log |\log (1-r)|}{|\log (1-r)|}
$$

is differentiable in $(0,1), \lim _{r \rightarrow 1^{-}} \sigma(r)=\sigma_{M}(f)$,

$$
\sigma^{\prime}(r)(1-r)|\log (1-r)|=\frac{1-\log |\log (1-r)|}{|\log (1-r)|} \rightarrow 0, \quad r \rightarrow 1^{-},
$$

and $(1-r)^{\sigma(r)} \log M(r, f)=1$ for all $0<r<1$. Hence $\sigma$ is a proximate order of $f$. Moreover,

$$
\frac{f^{\prime}(z)}{f(z)}=\frac{1}{(1-z)^{\alpha+1}}\left(\alpha \log \frac{1}{1-z}+1\right),
$$

and, in general, for all $k \in \mathbb{N}$,

$$
\frac{f^{(k)}(z)}{f(z)}=\left(\frac{1}{(1-z)^{\alpha+1}}\left(\alpha \log \frac{1}{1-z}+1\right)\right)^{k}+F(z),
$$

where $F \in A(\mathbb{D})$ satisfies

$$
|F(z)|=O\left(\frac{1}{(1-|z|)^{k(\alpha+1)-\alpha}}\left(\log \frac{1}{1-|z|}\right)^{k-1}\right) .
$$

Substituting $z=r$ and the constant $\alpha$ from (2.1) in (2.2), it follows that

$$
\frac{f^{(k)}(r)}{f(r)}=\left(\frac{1}{1-r}\right)^{(\sigma(r)+1) k}(\sigma(r)+o(1))+F(r),
$$


where

$$
|F(r)|=O\left(\frac{1}{1-r}\right)^{(\sigma(r)+1) k-\sigma(r)} .
$$

Therefore the assertion in Theorem 1.2 is sharp up to the logarithmic term in the case where $\sigma_{M}(f)>1$. Further, this also shows that ' $2(k-j)+\varepsilon$ ' cannot be replaced by '2 $(k-j)$ ' when $\sigma_{M}(f)=1$.

(b) For $0 \leq \alpha<1$, the function

$$
g(z)=\exp \left(\frac{1}{(1+z)^{\alpha}}-\frac{1}{1-z}\right)
$$

satisfies $\sigma_{M}(g)=\alpha$. Moreover,

$$
\frac{g^{\prime}(z)}{g(z)}=-\frac{\alpha}{(1+z)^{\alpha+1}}-\frac{1}{(1-z)^{2}},
$$

and, in general, for all $k \in \mathbb{N}$,

$$
\frac{g^{(k)}(z)}{g(z)}=\left(\frac{-1}{(1-z)^{2}}\right)^{k}+G(z)
$$

where $G \in A(\mathbb{D})$ satisfies

$$
|G(z)|=O\left(\left(\frac{1}{1-|z|}\right)^{\max \{(1+\alpha) k, 2 k-1\}}\right) .
$$

This shows that the constant 2 in Theorem 1.2 cannot be replaced by a smaller one when $0 \leq \sigma_{M}(g)<1$.

The discussion at the end of Section 1 in [13] shows that the equality in (1.7) can be attained. The sharpness of the remaining assertions in Theorem 1.4 is considered in the next example.

EXAMPLE 2. For $\beta \geq 1$ the functions

$$
f_{1}(z)=\exp \left(\frac{\beta}{1-z}\right)^{\beta} \text { and } f_{2}(z)=\exp \left(\frac{\beta}{1-z}\right)^{\beta+1}
$$

are linearly independent solutions of

$$
f^{\prime \prime}(z)+A_{1}(z) f^{\prime}(z)+A_{0}(z) f(z)=0,
$$

where

$$
A_{0}(z)=\frac{\beta^{\beta+1}(\beta+1)}{(1-z)^{\beta+2}(z+\beta)}+\frac{\beta^{2 \beta+2}(\beta+1)}{(1-z)^{2 \beta+3}}
$$

and

$$
A_{1}(z)=\frac{\beta^{\beta+1}(z-\beta-2)}{(1-z)^{\beta+2}}-\frac{(\beta+1)(z+\beta+1)}{(z+\beta)(1-z)}
$$


belong to $A(\mathbb{D})[13]$. Clearly $A_{0} \in G_{2 \beta+3}, A_{1} \in G_{\beta+2}$, and

$$
\sigma_{M}\left(f_{1}\right)=\beta=\min _{j=1,2}\left\{\frac{p_{0}-p_{j}}{j}-1\right\}
$$

which shows that the equality in (1.8) is attained for $f=f_{1}$. On the other hand, $n=1$ is the smallest index such that (1.11) holds, and

$$
\max _{j=0,1}\left\{\frac{p_{j}}{k-j}-1\right\}=\beta+1=\sigma_{M}\left(f_{2}\right)>\sigma_{M}\left(f_{1}\right) .
$$

This means that there is exactly one solution in the basis $\left\{f_{1}, f_{2}\right\}$ such that the equality in (1.12) is satisfied. Therefore the estimate for the number of linearly independent solutions of maximal growth is the best possible in this case.

The final example in this section shows that the assumption $\alpha \geq 1$ in Theorem 1.4(b) is necessary.

EXAMPLE 3. If $0<\alpha<1$, then the functions

$$
f_{1}(z)=\exp \left(\frac{1}{(1+z)^{\alpha}}-\frac{1}{1-z}\right) \text { and } f_{2}(z)=\exp \left(-\frac{1}{1-z}\right)
$$

are linearly independent solutions of (2.3), where

$$
A_{0}(z)=\frac{(\alpha-1) z^{2}-(1+2 \alpha) z+\alpha+4}{(1+z)(1-z)^{4}}+\frac{\alpha}{(1+z)^{1+\alpha}(1-z)^{2}}
$$

and

$$
A_{1}(z)=\frac{(1+\alpha) z^{2}-2 \alpha z+\alpha+3}{(1+z)(1-z)^{2}}+\frac{\alpha}{(1+z)^{1+\alpha}} .
$$

Clearly $A_{0} \in G_{4}, A_{1} \in G_{2}, \sigma_{M}\left(f_{1}\right)=\alpha$ and $\sigma_{M}\left(f_{2}\right)=0$. Therefore the assumption $\alpha \geq 1$ in Theorem 1.4(b) is necessary.

\section{Proof of Theorem 1.2}

3.1. Basic properties of proximate orders. We begin by recalling some basic properties of proximate orders; see [12, Section 1.6] and also [1, 17]. In what follows, a function $L:[0,1) \rightarrow \mathbb{R}_{+}$is called slowly varying if

$$
\lim _{r \rightarrow 1^{-}} \frac{L(1-\beta(1-r))}{L(r)}=1
$$

for all $\beta \in(0,1)$. 
LEMMA 3.1. Let $f \in A(\mathbb{D})$ be such that $\sigma_{M}(f) \in(0, \infty)$, and let $\sigma$ be a proximate order of $f$. Then the following assertions hold.

(a) The function $(1-r)^{-\sigma(r)+\sigma_{M}(f)}$ is slowly varying, and $(1-r)^{-\sigma(r)}$ is a monotonically increasing unbounded function on $\left[R_{0}, 1\right)$ for some $R_{0}>0$.

(b) If $\beta \in(0,1)$, then

$$
(\beta(1-r))^{-\sigma(1-\beta(1-r))}=(1+o(1)) \beta^{-\sigma_{M}(f)}(1-r)^{-\sigma(r)}, \quad r \rightarrow 1^{-} .
$$

(c) If $-1 \leq \lambda<\sigma_{M}(f)-1$ and $R_{0}>0$ is the constant in Definition 1.1(A), then

$$
\int_{r_{0}}^{r} \frac{d t}{(1-t)^{\sigma(t)-\lambda}}=\frac{1+o(1)}{\left(\sigma_{M}(f)-\lambda-1\right)(1-r)^{\sigma(r)-\lambda-1}}, \quad r \rightarrow 1^{-} .
$$

Proof. Assertion (a) is [12, Theorem 1.6.2], while assertion (c) is [12, Theorem 1.6.3]. Define $L(r)=(1-r)^{-\sigma(r)+\sigma_{M}(f)}$, which is slowly varying by (a). Then

$$
\frac{(\beta(1-r))^{-\sigma(1-\beta(1-r))}}{(1-r)^{-\sigma(r)}}=\frac{(\beta(1-r))^{-\sigma_{M}(f)} L(1-\beta(1-r))}{(1-r)^{-\sigma_{M}(f)} L(r)}=\frac{1+o(1)}{\beta^{\sigma_{M}(f)}},
$$

which proves (b).

LEMMA 3.2. Let $f \in A(\mathbb{D})$ be such that $\sigma_{M}(f) \in(0, \infty)$, and let $\sigma$ be a proximate order of $f$. Then $\sigma_{M}(f)=\sigma_{M}\left(f^{(m)}\right)$ and $\sigma$ is a proximate order of $f^{(m)}$ for any positive integer $m$.

PROOF. It suffices to prove the assertions for $m=1$. Cauchy's formula yields

$$
\left|f^{\prime}(z)\right| \leq \frac{M(R, f)}{2 \pi} \int_{|\zeta|=R} \frac{|d \zeta|}{|\zeta-z|^{2}}=\frac{R \cdot M(R, f)}{R^{2}-|z|^{2}}
$$

where $z \in \mathbb{D}$ is arbitrary and $R=(1+|z|) / 2$. It follows that $\sigma_{M}\left(f^{\prime}\right) \leq \sigma_{M}(f)$. Conversely,

$$
M(r, f) \leq|f(0)|+\int_{0}^{r} M\left(s, f^{\prime}\right) d s \leq|f(0)|+r M\left(r, f^{\prime}\right),
$$

so that $\sigma_{M}(f) \leq \sigma_{M}\left(f^{\prime}\right)$. Thus $\sigma_{M}(f)=\sigma_{M}\left(f^{\prime}\right)$. This calculation can easily be modified, using Lemma 3.1(b), to obtain condition (D) in Definition 1.1 for $f^{\prime}$. It follows that $\sigma$ is a proximate order of $f^{\prime}$.

3.2. An estimate for the minimum modulus. The minimum modulus of a function $f \in A(\mathbb{D})$ is defined by $\mu(r, f)=\min _{|z|=r}|f(z)|$. The following special case of an estimate due to Linden [17, Theorem 3] will play an important role in our reasoning.

Theorem A. Let $f \in A(\mathbb{D})$ and $\frac{1}{2} \leq \alpha<1$. Then there exists $R_{0}=R_{0}(\alpha) \in(0,1)$ such that for any $R \in\left[R_{0}, 1\right)$ there exists a set $E_{R} \subset\left[R^{2}, R\left(R+\frac{1}{16}(1-R)\right)\right]$ of 
measure at least $\frac{1}{32} R(1-R)$ such that

$$
\begin{aligned}
\log \mu(r, f) \geq- & \frac{C}{(1-R)^{1 / \alpha}} \log \frac{1}{1-R} \\
& \times\left(\int_{0}^{R} \log ^{+} M(t, f)(R-t)^{1 / \alpha-1} d t+\log ^{+} M\left(R_{0}, f\right)\right)
\end{aligned}
$$

for all $r \in E_{R}$ and for some constant $C=C\left(\alpha, R_{0}\right)>0$.

Throughout the rest of this section, suppose that $f \in A(\mathbb{D})$ with $\sigma_{M}(f) \in(1, \infty)$, and let $\sigma$ be a proximate order of $f$. We proceed to state and prove a consequence of Theorem A involving proximate order; see (3.7) below.

Let $\alpha \in\left[\frac{1}{2}, 1\right)$ be such that $\alpha^{-1}<\sigma_{M}(f)$. Let $R_{0}$ be the largest of the numbers $R_{0}$ in Definition 1.1(A), Lemma 3.1(a)(c) and Theorem A. Define $r_{v}=1-2^{-v}$, where $v$ is a nonnegative integer. Let $v$ be sufficiently large that $r_{v} \geq R_{0}$. Then there is a constant $C=C\left(R_{0}\right)>1$ such that

$$
\log ^{+} M(r, f) \leq C(1-r)^{-\sigma(r)}
$$

for all $r \in[0,1)$. Choose $R=r_{v+1}$ in Theorem A to obtain a set $E_{r_{v+1}}$,

$$
E_{r_{v+1}} \subset\left[r_{v+1}^{2}, r_{v+1}\left(r_{v+1}+16^{-1}\left(1-r_{v+1}\right)\right)\right] \subset\left(r_{v}, r_{v+1}\right),
$$

of measure at least

$$
32^{-1} r_{v+1}\left(1-r_{v+1}\right)=2^{-v-6}\left(1-2^{-v-1}\right) \geq 2^{-v-7}
$$

such that

$$
\begin{aligned}
\log \mu(r, f) \geq & -\frac{C\left(\alpha, R_{0}\right)}{\left(1-r_{v+1}\right)^{1 / \alpha}} \log \frac{1}{1-r_{v+1}} \\
& \times\left(\int_{0}^{r_{v+1}} \log ^{+} M(t, f)\left(r_{v+1}-t\right)^{1 / \alpha-1} d t+\log ^{+} M\left(R_{0}, f\right)\right)^{(3.5)}
\end{aligned}
$$

for all $r \in E_{r_{v+1}}$. If $r \in\left(r_{\nu}, r_{\nu+1}\right)$, then $1-r \leq 1-r_{\nu}=2\left(1-r_{\nu+1}\right)$, and so

$$
\frac{1}{\left(1-r_{\nu+1}\right)^{1 / \alpha}} \log \frac{1}{1-r_{\nu+1}} \leq \frac{2^{1 / \alpha}}{(1-r)^{1 / \alpha}} \log \frac{2}{1-r} \leq \frac{C\left(\alpha, R_{0}\right)}{(1-r)^{1 / \alpha}} \log \frac{1}{1-r} .
$$

Since $\alpha^{-1}-1>0$, the estimates (3.5), (3.6) and (3.2) yield

$$
\begin{aligned}
\log \mu(r, f) \geq- & \frac{C\left(\alpha, R_{0}\right)}{(1-r)^{1 / \alpha}} \log \frac{1}{1-r} \\
& \times\left(\int_{0}^{r_{\nu+1}}(1-t)^{1 / \alpha-1-\sigma(t)} d t+\left(1-R_{0}\right)^{-\sigma\left(R_{0}\right)}\right)
\end{aligned}
$$


for all $r \in E_{r_{v+1}}$. Since $\alpha^{-1}-1<\sigma_{M}(f)-1$, we may apply Lemma 3.1(b), with $\beta=\frac{1}{2}$, and Lemma 3.1(c), with $\lambda=\alpha^{-1}-1$, to obtain

$$
\begin{aligned}
\log \mu(r, f) & \geq-\frac{C\left(\alpha, \sigma_{M}(f), R_{0}\right)}{(1-r)^{1 / \alpha}} \frac{1}{\left(1-r_{\nu+1}\right)^{\sigma\left(r_{\nu+1}\right)-1 / \alpha}} \log \frac{1}{1-r} \\
& \geq-\frac{C\left(\alpha, \sigma_{M}(f), R_{0}\right)}{(1-r)^{\sigma(r)}} \log \frac{1}{1-r}
\end{aligned}
$$

for all $r \in E_{r_{v+1}}$. This estimate will be needed in proving Theorem 1.2.

3.3. An estimate for the zeros. Let $n(\zeta, h, f)$ denote the number of zeros of the function $f \in A(\mathbb{D})$ in the closed disc $\bar{D}(\zeta, h)=\{w:|\zeta-w| \leq h\}$. Replacing $z$ by $R z$ in [17, Theorem 2], we obtain the following result.

Theorem B. Suppose that $f \in A(\mathbb{D})$ and $0<R<1$. For all $\alpha \in\left[\frac{1}{2}, 1\right)$ there exists $R_{0}=R_{0}(\alpha) \in(0,1)$ such that, for all $\zeta \in \mathbb{D}$ satisfying $|\zeta|=r<R$,

$$
n(\zeta, h, f) \leq \frac{C(\alpha, \eta)}{(R-r)^{1 / \alpha}}\left(\int_{0}^{R} \log ^{+} M(t, f)(R-t)^{1 / \alpha-1} d t+\log ^{+} M\left(R_{0}, f\right)\right),
$$

where $h=(\eta / R)(R-r)$ and $0<\eta<\frac{1}{6}$.

Suppose now that $f \in A(\mathbb{D})$ with $\sigma_{M}(f) \in(1, \infty)$, let $\alpha \in\left[\frac{1}{2}, 1\right)$ be such that $\alpha^{-1}<\sigma_{M}(f)$, and let $\sigma$ be a proximate order of $f$. We proceed to state and prove a consequence of Theorem B involving proximate order; see (3.10) below.

Let $v$ be sufficiently large that $r_{v} \geq R_{0}$, where $r_{v}=1-2^{-v}$ and $R_{0}$ is the constant from Section 3.2. Let $r \in\left[r_{v}, r_{v+1}\right)$. Using Lemma 3.1(b), with $\beta=\frac{1}{4}$, and Lemma 3.1(c), with $\lambda=\alpha^{-1}-1$, we obtain

$$
\int_{0}^{r_{\nu+2}}(1-t)^{1 / \alpha-1-\sigma(t)} d t \leq C\left(\alpha, \sigma_{M}(f), R_{0}\right)(1-r)^{1 / \alpha-\sigma(r)} .
$$

Choose $R=2 r /(r+1)$, where $r \in\left[r_{v}, r_{v+1}\right)$. Then $R \in\left(r_{v}, r_{v+2}\right)$. The estimates in (3.8) and (3.9) yield

$$
n\left(\zeta, \frac{\eta(1-r)}{2}, f\right) \leq \frac{C\left(\alpha, \eta, \sigma_{M}(f), R_{0}\right)}{(1-r)^{\sigma(r)}}
$$

for all $\zeta \in \mathbb{D}$ satisfying $|\zeta|<2 r /(r+1)$ and for all $0<\eta<1 / 6$.

Define $\mathcal{A}_{1}=\bar{D}\left(0, \frac{1}{2}\right)$ and the annuli $\mathcal{A}_{v}=\left\{\zeta: r_{v-1}<|\zeta| \leq r_{v}\right\}$ for $v \geq 2$. Then clearly $\mathbb{D}=\bigcup_{\nu} \mathcal{A}_{\nu}$. Based on (3.10), we now state and prove an estimate which is crucial in proving Theorem 1.2.

LEMMA 3.3. Let $f \in A(\mathbb{D})$ be such that $\sigma_{M}(f) \in(1, \infty)$, and let $\sigma$ be a proximate order of $f$. Let $\left\{a_{k}\right\}$ denote the sequence of zeros of $f$ listed according to multiplicities and ordered by increasing modulus, and let $0<\delta<1$. Then there exists a countable 


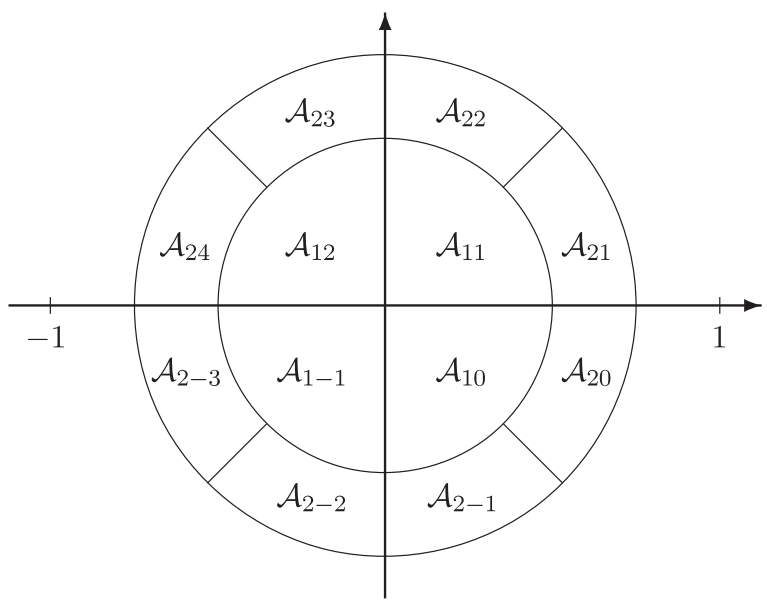

FIgURE 1 . The cases $s=1$ and $s=2$.

collection of discs $D_{v j}=\left\{w:\left|w-z_{v j}\right|<\rho_{v j}\right\}$, where $\rho_{v j}<1-\left|z_{v j}\right|$, such that, for all $z \in \mathcal{A}_{v} \backslash \bigcup_{j} D_{v j}$,

$$
\sum_{\left|a_{k}\right| \leq r_{\nu+1}} \frac{1}{\left|z-a_{k}\right|} \leq \frac{C\left(\alpha, \sigma_{M}(f), R_{0}\right)}{\delta(1-r)^{\sigma(r)+1}} \log \frac{1}{1-r},
$$

where

$$
\sum_{R<\left|z_{v j}\right|<1} \rho_{\nu j} \leq \delta(1-R), \quad R \rightarrow 1^{-} .
$$

PROOF. Without loss of generality, we may assume that $\arg z=0$. For $s \in \mathbb{N}$ define $I_{s}=\left\{-2^{s}+1, \ldots,-1,0,1, \ldots, 2^{s}\right\}$. For $\tau \in I_{s}$, define the polar rectangles

$$
\mathcal{A}_{s \tau}=\left\{\zeta \in \mathcal{A}_{s}:(\tau-1) \pi 2^{-s} \leq \arg \zeta<\tau \pi 2^{-s}\right\}
$$

see Figure 1 for an illustration.

Denote

$$
I_{s}^{*}= \begin{cases}I_{s} & \text { if } 1 \leq s \leq v-2 \\ I_{s} \backslash\{0,1\} & \text { if } v-1 \leq s \leq v+1 .\end{cases}
$$

With this notation, we have the following result.

Lemma 3.4. Let $v \geq 2,2 \leq s \leq v+1, \tau \in I_{s}^{*}, \zeta \in \mathcal{A}_{s \tau}, z \in \mathcal{A}_{v}$ and $\arg z=0$. Then

$$
|\zeta-z| \geq \begin{cases}\tau 2^{-s-1} & \text { if } \tau>0 \\ (|\tau|+1) 2^{-s-1} & \text { if } \tau \leq 0\end{cases}
$$




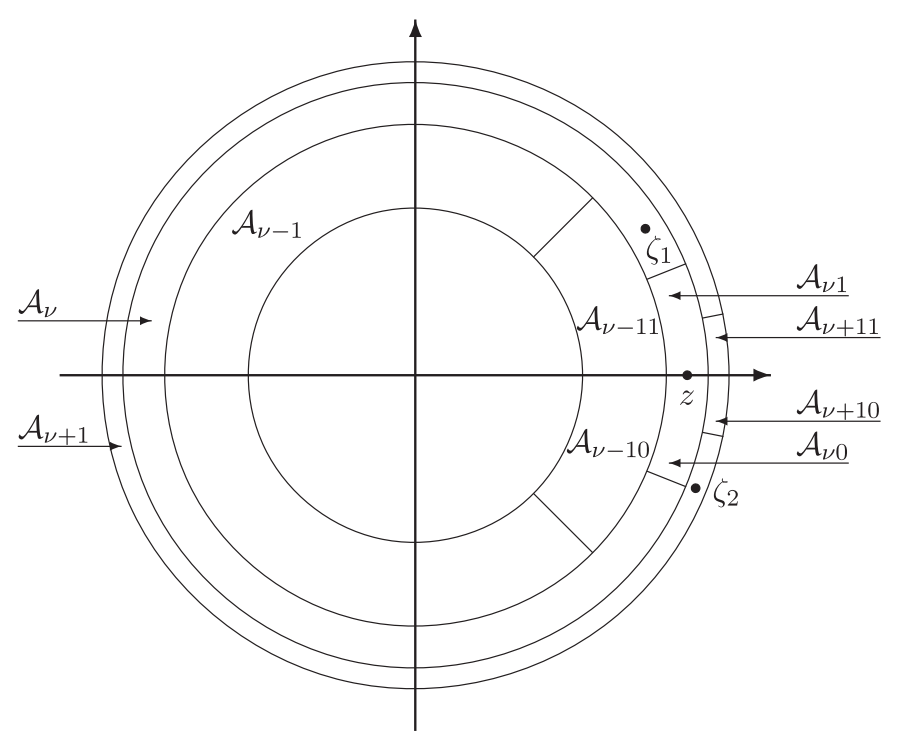

FIgURE 2. Illustration of Lemma 3.4. The $\mathcal{A}$-sets in the left half-plane are annuli, while the $\mathcal{A}$-sets in the right half-plane are polar rectangles.

PROOF. Suppose first that $|\arg \zeta| \leq \pi / 2$. Then $-2^{s-1}+1 \leq \tau \leq 2^{s-1}$ by definition of the sets $\mathcal{A}_{s \tau}$. The point $\zeta=\zeta_{1}$ in Figure 2 illustrates the case where $0<\tau \leq 2^{s-1}$, while the point $\zeta=\zeta_{2}$ illustrates the case where $-2^{s-1}+1 \leq \tau \leq 0$.

Since $\zeta \in \mathcal{A}_{s \tau}, z \in \mathcal{A}_{v}$ and $\arg z=0$,

$$
|\zeta-z| \geq|\zeta| \sin (\arg \zeta) \geq r_{s-1} \sin \left(\frac{\tau-1}{2^{s} \pi}\right) \geq \frac{\tau-1}{2^{s-1}} r_{s-1}
$$

for all $\tau>0$, and

$$
|\zeta-z| \geq|\zeta| \sin (|\arg \zeta|) \geq r_{s-1} \sin \left(\frac{|\tau|}{2^{s}} \pi\right) \geq \frac{|\tau|}{2^{s-1}} r_{s-1}
$$

for all $\tau \leq 0$. Thus

$$
|\zeta-z| \geq \begin{cases}\frac{\tau-1}{2^{s-1}} r_{s-1} & \text { if } \tau>0 \\ \frac{|\tau|}{2^{s-1}} r_{s-1} & \text { if } \tau \leq 0\end{cases}
$$

If $2 \leq s \leq v-2$, we have in addition that

$$
|\zeta-z| \geq|z|-\operatorname{Re} \zeta \geq r_{\nu-1}-r_{s}=2^{-s}-2^{1-v} \geq 2^{-s}-2^{-s-1}=2^{-s-1} .
$$

Clearly, $r_{s-1}=1-2^{-s+1} \geq \frac{1}{2}$ for $s \geq 2$, while $(\tau-1) / \tau \geq \frac{1}{2}$ for $\tau \geq 2$ whereas $|\tau| /(|\tau|+1) \geq \frac{1}{2}$ for $\tau \leq-1$. Therefore applying (3.13) for $\tau \notin\{0,1\}$, and (3.14) for $\tau \in\{0,1\}$, we prove Lemma 3.4 in the case where $|\arg \zeta| \leq \pi / 2$. 
Suppose then that $\pi / 2 \leq|\arg \zeta| \leq \pi$. In this case either $2^{s-1}<\tau \leq 2^{s}$ or $-2^{s}+$ $1 \leq \tau<-2^{s-1}+1$, and therefore

$$
\frac{1}{2} \geq \begin{cases}\tau 2^{-s-1} & \text { if } \tau>0 \\ (|\tau|+1) 2^{-s-1} & \text { if } \tau \leq 0 .\end{cases}
$$

The statement of Lemma 3.4 in the case where $\pi / 2 \leq|\arg \zeta| \leq \pi$ now follows by the inequality $|\zeta-z| \geq \frac{1}{2}$, which is valid since $\arg z=0$ and $s \geq 2$.

We return to the proof of Lemma 3.3. Let $n(U)=n\left(U,\left\{a_{k}\right\}\right)$ denote the number of the points $a_{k}$ in the set $U \subset \mathbb{D}$. Let

$$
L(r, \varphi)=\left\{\zeta: r \leq|\zeta| \leq \frac{1+r}{2},|\arg \zeta-\varphi| \leq \frac{\pi}{4}(1-r)\right\}
$$

and denote

$$
n_{1}(r)=n_{1}\left(r,\left\{a_{k}\right\}\right)=\max _{0 \leq \varphi \leq 2 \pi} n(L(r, \varphi)) .
$$

If $z \in \mathcal{A}_{v}$ and $z \neq a_{k}$ for all $k$, write

$$
\begin{aligned}
\sum_{\left|a_{k}\right| \leq r_{\nu+1}} \frac{1}{\left|z-a_{k}\right|} & =\sum_{s=1}^{\nu+1} \sum_{\tau \in I_{s}} \sum_{a_{k} \in \mathcal{A}_{s \tau}} \frac{1}{\left|z-a_{k}\right|} \\
& \leq \sum_{s=1}^{\nu+1} \sum_{\tau \in I_{s}^{*}} \sum_{a_{k} \in \mathcal{A}_{s \tau}} \frac{1}{\left|z-a_{k}\right|}+\sum_{s=\nu-1}^{\nu+1} \sum_{\tau=0}^{1} \sum_{a_{k} \in \mathcal{A}_{s \tau}} \frac{1}{\left|z-a_{k}\right|} \\
& =S_{1}+S_{2} .
\end{aligned}
$$

To deal with the sum $S_{1}$ in (3.15), we first observe that $r_{s}=\left(1+r_{s-1}\right) / 2$ and $\tau \pi 2^{-s}-(\tau-1) \pi 2^{-s}=\pi 2^{-s}=2 \pi 2^{-2}\left(1-r_{s-1}\right)$. Lemma 3.4 now yields

$$
\begin{aligned}
S_{1} & \leq \sum_{s=1}^{\nu+1} \sum_{\tau \in I_{s}^{*}} \frac{n\left(\mathcal{A}_{s \tau}\right)}{\inf _{\zeta \in \mathcal{A}_{s \tau}|z-\zeta|}} \\
& \leq \sum_{s=1}^{v+1}\left(\sum_{\tau \in I_{s}^{*}, \tau>0} \frac{n_{1}\left(r_{s-1}\right)}{\tau} 2^{s+1}+\sum_{\tau \in I_{s}^{*}, \tau \leq 0} \frac{n_{1}\left(r_{s-1}\right)}{|\tau|+1} 2^{s+1}\right) \\
& =\sum_{s=1}^{v-2}\left(\sum_{\tau=1}^{2^{s}} \frac{n_{1}\left(r_{s-1}\right)}{\tau} 2^{s+2}\right)+\sum_{s=v-1}^{v+1}\left(\sum_{\tau=2}^{2^{s}} \frac{n_{1}\left(r_{s-1}\right)}{\tau} 2^{s+2}\right) \\
& \leq 8 \sum_{s=1}^{v+1} \frac{n_{1}\left(r_{s-1}\right)}{1-r_{s-1}}\left(\sum_{\tau=1}^{2^{s}} \frac{1}{\tau}\right) \leq 8 \sum_{\tau=1}^{2^{v+1}} \frac{1}{\tau} \cdot \sum_{s=1}^{v+1} \frac{n_{1}\left(r_{s-1}\right)}{1-r_{s-1}} \\
& \leq 8(1+(v+1) \log 2) \sum_{s=1}^{v+1} \frac{n_{1}\left(r_{s-1}\right)}{1-r_{s-1}} \leq 24 v \sum_{s=1}^{v+1} \frac{n_{1}\left(r_{s-1}\right)}{1-r_{s-1}} .
\end{aligned}
$$


For any $r \in(0,1)$ the set $L(r, \varphi)$ can be covered by a uniformly bounded number of discs $D(\zeta,(1-r) / 20)$, where $r \leq|\zeta| \leq(1+r) / 2$. Hence, by applying (3.10), it follows that

$$
\begin{aligned}
S_{1} & \leq 24 v \sum_{s=1}^{v+1} \frac{n_{1}\left(r_{s-1}\right)}{1-r_{s-1}}=24 v \sum_{s=0}^{\nu} \frac{n_{1}\left(r_{s}\right)}{1-r_{s}} \\
& \leq C\left(\alpha, \sigma_{M}(f), R_{0}\right) v \sum_{s=0}^{\nu} \frac{1}{\left(1-r_{s}\right)^{\sigma\left(r_{s}\right)+1}} \\
& \leq C\left(\alpha, \sigma_{M}(f), R_{0}\right) \log \frac{1}{1-r_{v}} \int_{0}^{r_{v+1}} \frac{d t}{(1-t)^{\sigma(t)+2}} .
\end{aligned}
$$

A reasoning similar to that leading to (3.7), using Lemma 3.1(c), with $\lambda=-1$, finally gives

$$
S_{1} \leq \frac{C\left(\alpha, \sigma_{M}(f), R_{0}\right)}{\left(1-r_{v}\right)^{\sigma\left(r_{v}\right)+1}} \log \frac{1}{1-r_{v}} .
$$

To deal with the sum $S_{2}$ in (3.15), define

$$
U=\bigcup_{s=\nu-1}^{\nu+1} \bigcup_{\tau=0}^{1} \mathcal{A}_{s \tau}, \quad N_{\nu}=n(U) \quad \text { and } \quad \delta_{\nu}=\delta \cdot 2^{-\nu-6}
$$

Then, by the Cartan lemma [16, pp. 19-21], there exists a finite collection of discs $D\left(w_{v j}, h_{v j}\right)$ with $\sum_{j} h_{v j}=2 \delta_{v}$ and a permutation of $\left\{a_{k}\right\} \subset U$, say $b_{1}, \ldots, b_{N_{v}}$, such that $\left|z-b_{m}\right|>m \delta_{v} / N_{v}$ for all $m=1, \ldots, N_{v}$ and $z \notin \bigcup_{j} D\left(w_{v j}, h_{v j}\right)$. Hence, by noting that

$$
n\left(\mathcal{A}_{s \tau}\right) \leq n_{1}\left(r_{s-1}\right), \quad s=v-1, \ldots, v+1, \tau=0,1,
$$

it follows that

$$
\begin{aligned}
S_{2} & =\sum_{a_{k} \in U} \frac{1}{\left|z-a_{k}\right|}=\sum_{m=1}^{N_{v}} \frac{1}{\left|z-b_{m}\right|} \leq \frac{N_{v}}{\delta_{v}} \sum_{m=1}^{N_{v}} \frac{1}{m} \\
& \leq \frac{2^{5} N_{v}}{\delta\left(1-r_{v+1}\right)}\left(1+\log N_{v}\right) \leq \frac{C}{\delta} \sum_{s=v-2}^{v+1} \frac{n_{1}\left(r_{s}\right)}{1-r_{s}} \log n_{1}\left(r_{s}\right) \\
& \leq \frac{C}{\delta\left(1-r_{v}\right)^{\sigma\left(r_{v}\right)+1}} \log \frac{1}{1-r_{v}}
\end{aligned}
$$

for all $z \in \mathcal{A}_{v}$ satisfying $z \notin \bigcup_{j} D\left(w_{v j}, h_{v j}\right)$.

By combining (3.15)-(3.17) and Lemma 3.1(b), with $\beta=\frac{1}{2}$, we conclude that

$$
\sum_{\left|a_{k}\right| \leq r_{v+1}} \frac{1}{\left|z-a_{k}\right|} \leq \frac{C\left(\alpha, \sigma_{M}(f), R_{0}\right)}{\delta(1-r)^{\sigma(r)+1}} \log \frac{1}{1-r}
$$

for all $z \in \mathcal{A}_{v}$ satisfying $z \notin \bigcup_{j} D\left(w_{v j}, h_{v j}\right)$. 
It remains to study the size of this exceptional set. For each $v$ we take into account only those discs $D\left(w_{v j}, h_{v j}\right)$ which intersect the annulus $\mathcal{A}_{v}$, and discard the rest of the discs. Each $v$ is then associated with a collection of discs $D_{v j}=D\left(z_{v j}, \rho_{v j}\right)$ such that

$$
r_{v-1}-\delta \cdot 2^{-v-5} \leq\left|z_{v j}\right| \leq r_{v}+\delta \cdot 2^{-v-5} .
$$

In particular, $z_{v j} \in \mathcal{A}_{v-1} \cup \mathcal{A}_{v} \cup \mathcal{A}_{v+1}$. Moreover, by the second inequality in (3.19),

$$
\left|z_{v j}\right|+\rho_{\nu j} \leq\left|z_{v j}\right|+\sum_{j} \rho_{\nu j} \leq\left|z_{v j}\right|+2 \delta_{v} \leq 1-2^{-v}+\delta \cdot 2^{-v-4}<1,
$$

which shows that all of the discs $D_{v j}$ are contained in $\mathbb{D}$. If now $R \in\left[r_{v-1}, r_{v}\right)$, we obtain

$$
\sum_{R \leq\left|z_{v j}\right|<1} \rho_{\nu j} \leq \sum_{s=v-1}^{\infty} \sum_{j} \rho_{s j}=2 \delta \sum_{s=v-1}^{\infty} 2^{-s-6}=\delta 2^{-v-3}<\delta(1-R) .
$$

This discussion together with (3.18) completes the proof of Lemma 3.3.

3.4. Proof of the case where $\sigma_{M}(f)>1$. Let $\sigma_{M}(f)>1$, and suppose first that $k=1$ and $j=0$. Denote $z=r e^{i \varphi}$, and let $0<r<R<1$. By the differentiated Poisson-Jensen formula,

$$
\left|\frac{f^{\prime}(z)}{f(z)}\right| \leq \frac{R}{\pi} \int_{0}^{2 \pi} \frac{|\log | f\left(R e^{i \theta}\right)|| d \theta}{\left|R e^{i \theta}-z\right|^{2}}+\sum_{\left|a_{k}\right| \leq R}\left(\frac{1}{\left|z-a_{k}\right|}+\frac{\left|a_{k}\right|}{\left|R^{2}-\bar{a}_{k} z\right|}\right)
$$

where $\left\{a_{k}\right\}$ is the sequence of zeros of $f$ listed according to multiplicities and ordered by increasing moduli. For any $\left|a_{k}\right| \leq R$,

$$
\frac{\left|a_{k}\right|}{\left|R^{2}-\bar{a}_{k} z\right|}=\frac{\left|a_{k}\right|}{R\left|z-a_{k}\right|} \cdot\left|\frac{\frac{a_{k}}{R}-\frac{z}{R}}{1-\frac{\overline{a_{k}}}{R} \cdot \frac{z}{R}}\right| \leq \frac{1}{\left|z-a_{k}\right|} .
$$

Fix $v$ large enough so that $r_{v-1} \geq R_{0}$. Let $z \in \mathcal{A}_{v} \backslash \bigcup_{j} D_{v j}$, where $\left\{D_{v j}\right\}$ is the family of discs found in Section 3.3. Choose $R \in E_{r_{v+2}}$, where $E_{r_{v+2}} \subset\left(r_{v+1}, r_{v+2}\right)$ is a set of measure at least $2^{-v-8}$ by (3.3) and (3.4). By (3.20), (3.21), Definition 1.1(D), (3.7) and Lemma 3.3, we obtain

$$
\begin{aligned}
\left|\frac{f^{\prime}(z)}{f(z)}\right| & \leq \frac{C\left(\alpha, \sigma_{M}(f), R_{0}\right)|\log (1-R)|}{(1-R)^{\sigma(R)}} \int_{0}^{2 \pi} \frac{d \theta}{\left|R e^{i \theta}-z\right|^{2}}+\sum_{\left|a_{k}\right| \leq r_{v+2}} \frac{2}{\left|z-a_{k}\right|} \\
& \leq \frac{C\left(\alpha, \sigma_{M}(f), R_{0}\right)|\log (1-R)|}{(R-r)(1-R)^{\sigma(R)}}+\frac{C\left(\alpha, \sigma_{M}(f), R_{0}\right)|\log (1-r)|}{\delta(1-r)^{\sigma(r)+1}} .
\end{aligned}
$$

Finally, since $R-r \geq r_{v+1}-r_{v}=1-r_{v+1}$ and

$$
1-r \leq 1-r_{\nu-1}=2\left(1-r_{\nu}\right)=8\left(1-r_{\nu+2}\right) \leq 8(1-R) \leq 8(1-r),
$$


an application of assertions (a) and (b) in Lemma 3.1, with $\beta=\frac{1}{8}$, yields

$$
\left|\frac{f^{\prime}(z)}{f(z)}\right| \leq \frac{C\left(\alpha, \sigma_{M}(f), R_{0}\right)}{\delta(1-r)^{\sigma(r)+1}} \log \frac{1}{1-r}
$$

This completes the proof of Theorem 1.2 in the case where $\sigma_{M}(f)>1, k=1$ and $j=0$.

Suppose then that $k$ and $j$ are integers satisfying $k>j \geq 0$. By applying Lemma 3.2 and the reasoning above to the functions $f^{(m)}, m=j, \ldots, k-1$, we get, for all $z \in \mathbb{D} \backslash \bigcup_{v} D_{v}^{(m)}$,

$$
\left|\frac{f^{(m+1)}(z)}{f^{(m)}(z)}\right| \leq \frac{C\left(\alpha, m, \sigma_{M}(f), R_{0}\right)}{\delta(1-r)^{\sigma(r)+1}} \log \frac{1}{1-r}
$$

where $D_{v}^{(m)}=D\left(z_{v}^{(m)}, \rho_{v}^{(m)}\right)$ and

$$
\sum_{R \leq\left|z_{v}^{(m)}\right|<1} \rho_{\nu}^{(m)}<\frac{\delta}{k-j}(1-R) .
$$

Therefore, for all $z \in \mathbb{D} \backslash \bigcup_{m=j}^{k-1} \bigcup_{v} D_{v}^{(m)}$,

$$
\left|\frac{f^{(k)}(z)}{f^{(j)}(z)}\right|=\prod_{m=j}^{k-1}\left|\frac{f^{(m+1)}(z)}{f^{(m)}(z)}\right| \leq C\left(\alpha, \sigma_{M}(f), j, k, R_{0}\right)\left(\frac{|\log (1-r)|}{\delta(1-r)^{\sigma(r)+1}}\right)^{k-j}
$$

where

$$
\sum_{m=j}^{k-1} \sum_{R \leq \mid z_{v}^{(m)}} \rho_{v}^{(m)}<\delta(1-R)
$$

This completes the proof of Theorem 1.2 in the case where $\sigma_{M}(f)>1$.

3.5. Proof of the case where $\sigma_{M}(f) \leq 1$. Suppose first that $0<\sigma_{M}(f) \leq 1$. It is easy to see that the integral

$$
\int_{0}^{1} \frac{d t}{(1-t)^{\sigma(t)+1-1 / \alpha}}
$$

converges for any fixed $\alpha \in\left[\frac{1}{2}, 1\right)$. Then following the reasoning in Sections 3.2 and 3.3 , we obtain the estimates

$$
\log \mu(r, f) \geq-\frac{C\left(\alpha, R_{0}\right)}{(1-r)^{1 / \alpha}} \log \frac{1}{1-r}
$$

and

$$
n(\zeta, \eta(1-r) / 2, f) \leq \frac{C\left(\alpha, \eta, R_{0}\right)}{(1-r)^{1 / \alpha}}
$$


corresponding to (3.7) and (3.10), respectively. The rest of the proof in the case where $0<\sigma_{M}(f) \leq 1$ repeats that in the case where $\sigma_{M}(f)>1$ but uses the estimates just obtained instead of (3.7) and (3.10). In particular, the relevant analogue of (3.11) for $f$ satisfying $0<\sigma(f) \leq 1$ is given by

$$
\sum_{\left|a_{k}\right| \leq r_{v+1}} \frac{1}{\left|z-a_{k}\right|} \leq \frac{C\left(\alpha, R_{0}\right)}{\delta(1-r)^{2+\varepsilon}}
$$

where $\varepsilon>0$ and other quantities are as in Lemma 3.3. The details are omitted.

Suppose then that $\sigma_{M}(f)=0$. It follows by [3, Theorem 3.1 and Corollary 3.2] that there exists a countable collection of discs $D_{v}=\left\{w:\left|w-z_{\nu}\right|<r_{\nu}\right\} \subset \mathbb{D} \backslash\{0\}$ satisfying

$$
\sum_{\nu=1}^{\infty} \frac{r_{\nu}}{1-\left|z_{\nu}\right|}<\infty
$$

and a constant $\rho \in(0,1)$ such that

$$
\left|\frac{f^{(k)}(z)}{f^{(j)}(z)}\right| \leq\left(\frac{1}{1-|z|}\right)^{2(k-j)+\varepsilon}
$$

for all $z$ satisfying $z \in \mathbb{D} \backslash \bigcup_{v} D_{v}$ and $\rho<|z|<1$. Note that since $D_{v} \subset \mathbb{D}$, we have $r_{v}<1-\left|z_{v}\right|$.

We may suppose that the sequence $\left\{z_{v}\right\}$ is organized by increasing moduli. Hence there exists a positive integer $N_{0}$ such that

$$
\sum_{\nu=N_{0}}^{\infty} \frac{r_{\nu}}{1-\left|z_{v}\right|}<\delta
$$

Let $\max \left\{\rho,\left|z_{N_{0}}\right|\right\} \leq R<1$. Now

$$
\sum_{R<\left|z_{v}\right|<1} r_{v} \leq(1-R) \sum_{R<\left|z_{v}\right|<1} \frac{r_{v}}{1-\left|z_{v}\right|} \leq \delta(1-R) .
$$

This completes the proof of Theorem 1.2 in the case where $\sigma_{M}(f)=0$.

\section{Proof of Theorem 1.4}

4.1. Proof of (a). Inequality (1.7) is proved in [13, Theorem 1]. To obtain (1.8), the proof of [3, Theorem 2.3] is followed. We need the following auxiliary result, which shows that the growth of a $G_{p}$-function is near maximal (see (1.6)) on a relatively large set. The assertion can be proved similarly to [3, Lemma 5.1] or [4, Lemma 9]. See also [2, Lemma 3] and [13, Lemma 2] for similar results.

Lemma 4.1. Suppose that $A \in G_{p}$ for some $p \in(0, \infty)$, and let $\varepsilon, \eta \in(0,1)$. Then there is a set $F \subset[0,1)$ with $\bar{D}(F) \geq \eta$ such that

$$
\liminf _{\substack{r \rightarrow 1^{-} \\ r \in F}} \frac{\log ^{+} M(r, A)}{|\log (1-r)|} \geq p-\varepsilon
$$


Let $\varepsilon, \delta \in\left(0, \frac{1}{2}\right)$. By Corollary 1.3 there exists an exceptional set $E \subset[0,1)$ with $\bar{D}(E) \leq 2 \delta$ such that for any $z \in \mathbb{D}$ satisfying $|z| \notin E$,

$$
\left|A_{0}(z)\right| \leq \sum_{j=1}^{k}\left|A_{j}(z)\right|\left|\frac{f^{(j)}(z)}{f(z)}\right| \leq \sum_{j=1}^{k}\left(\frac{1}{1-|z|}\right)^{p_{j}+j\left(\max \left\{\sigma_{M}(f), 1\right\}+1+\varepsilon\right)},
$$

where $A_{k}(z) \equiv 1$ and $p_{k}=0$. By applying Lemma 4.1 to the function $A_{0}$ in (4.1), it follows that

$$
p_{0}-\varepsilon \leq \max _{1 \leq j \leq k}\left\{p_{j}+j\left(\max \left\{\sigma_{M}(f), 1\right\}+1+\varepsilon\right)\right\} .
$$

By rearranging terms and letting $\varepsilon \rightarrow 0^{+}$in (4.2), we obtain (1.8).

4.2. Proof of (c). If $n=0$ is the smallest index such that (1.11) holds, then

$$
\frac{p_{0}-p_{j}}{j}=\frac{p_{0}}{j}-\frac{p_{j}}{k-j} \cdot \frac{k-j}{j} \geq \frac{p_{0}}{j}-\frac{p_{0}}{k} \cdot \frac{k-j}{j}=\frac{p_{0}}{k}
$$

for all $j=1, \ldots, k-1$, and so the minimum in (1.8) is attained when $j=k$. Thus, by (1.8) and (1.9),

$$
\sigma_{M}(f) \geq \frac{p_{0}}{k}-1
$$

for all nontrivial solutions $f$ of (1.5). Therefore, by (1.7) and (4.4), there are $k$ linearly independent solutions $f$ for which (1.12) is valid.

Suppose then that $n \geq 1$. In this part of the proof, the standard method of order reduction will be used. The set $E \subset[0,1)$ from now on is not necessarily the same at each occurrence, but it always satisfies $\bar{D}(E) \leq \delta<1$. For simplicity, a finite union of such sets is denoted again by $E$. The related constants $\delta$ will be chosen such that their sum, denoted again by $\delta$, satisfies $\delta<1$.

We begin with the following consequence of Corollary 1.3.

COROLlary 4.2. Let $g, h \in A(\mathbb{D})$ be such that

$$
\max \left\{\sigma_{M}(g), \sigma_{M}(h)\right\} \leq \beta<\infty .
$$

Let $n \geq 1$ be an integer, and let $\delta, \varepsilon \in(0,1)$. Denote $f=g / h$. Then there exists an exceptional set $E \subset[0,1)$ with $\bar{D}(E) \leq \delta$ such that

$$
\left|\frac{f^{(n)}(z)}{f(z)}\right| \leq\left(\frac{1}{1-|z|}\right)^{(\max \{\beta, 1\}+1) n+\varepsilon}
$$

for all $z \in \mathbb{D}$ satisfying $|z| \notin E$.

PROOF. By calculating

$$
f^{\prime}=\frac{g^{\prime} h-g h^{\prime}}{h^{2}}, \quad f^{\prime \prime}=\frac{\left(g^{\prime \prime} h-g h^{\prime \prime}\right) h^{2}-\left(g^{\prime} h-g h^{\prime}\right) 2 h h^{\prime}}{h^{4}}, \ldots,
$$


we see, by means of Lemma 3.2, that for any $k \in\{1, \ldots, n\}$ there exist functions $g_{k}, h_{k} \in A(\mathbb{D})$ such that $\max \left\{\sigma_{M}\left(g_{k}\right), \sigma_{M}\left(h_{k}\right)\right\} \leq \beta$ and $f^{(k)}=g_{k} / h_{k}$. Since $f^{\prime} / f=$ $g^{\prime} / g-h^{\prime} / h$, assertion (4.5) for $n=1$ follows by Corollary 1.3. Suppose then that (4.5) is valid for $n=j$. Clearly

$$
\frac{f^{(j+1)}}{f}=\frac{f^{(j+1)}}{f^{(j)}} \cdot \frac{f^{(j)}}{f}=\frac{h_{j}}{g_{j}} \cdot\left(\frac{g_{j}}{h_{j}}\right)^{\prime} \cdot \frac{f^{(j)}}{f}=\left(\frac{g_{j}^{\prime}}{g_{j}}-\frac{h_{j}^{\prime}}{h_{j}}\right) \cdot \frac{f^{(j)}}{f} .
$$

The case $n=j+1$ now follows from (4.6) by applying Corollary 1.3 to the functions $g_{j}, h_{j}$ and the inductive assumption to the logarithmic derivative $f^{(j)} / f$.

The following two lemmas are obtained by modifying the analogous plane results in [7, Section 6].

LEMMA 4.3. Let $f_{0,1}, \ldots, f_{0, m}$ be $m \geq 2$ linearly independent analytic solutions of

$$
f^{(k)}(z)+A_{0, k-1}(z) f^{(k-1)}(z)+\cdots+A_{0,1}(z) f^{\prime}(z)+A_{0,0}(z) f(z)=0,
$$

where $k \geq m$ and $A_{0,0}, \ldots, A_{0, k-1} \in A(\mathbb{D})$. For $1 \leq n \leq m-1$, set

$$
f_{n, j}=\left(\frac{f_{n-1, j+1}}{f_{n-1,1}}\right)^{\prime}, \quad j=1, \ldots, m-n .
$$

Then $f_{n, 1}, \ldots, f_{n, m-n}$ are linearly independent meromorphic solutions of

$$
f^{(k-n)}(z)+A_{n, k-n-1}(z) f^{(k-n-1)}(z)+\cdots+A_{n, 1}(z) f^{\prime}(z)+A_{n, 0}(z) f(z)=0,
$$

where

$$
A_{n, j}(z)=\sum_{l=j+1}^{k-n+1}\left(\begin{array}{c}
l \\
j+1
\end{array}\right) A_{n-1, l}(z) \frac{f_{n-1,1}^{(l-j-1)}(z)}{f_{n-1,1}(z)}
$$

for $j=0, \ldots, k-1-n$. Here $A_{j, k-j}(z) \equiv 1$ for all $j=0, \ldots, n$.

Moreover, let $\varepsilon>0$, and suppose that for each $j \in\{0, \ldots, k-1\}$ there exists a $p_{j} \geq 0$ such that $A_{0, j} \in G_{p_{j}}$. Set

$$
M_{p}=\max _{p \leq l \leq k-1}\left\{p_{l}+(l-p)(\max \{\beta, 1\}+1)\right\},
$$

where $\beta=\max _{1 \leq l \leq m}\left\{\sigma_{M}\left(f_{0, l}\right)\right\}<\infty$. Then

$$
M\left(r, A_{n, j}\right) \leq\left(\frac{1}{1-r}\right)^{M_{n+j}+\varepsilon}
$$

for all $r \in[0,1) \backslash E$ and $j=0, \ldots, k-1-n$.

PROOF. By the proof of [7, Lemma 6.4], the functions $f_{n, j}$ in (4.7) are linearly independent meromorphic solutions of (4.8), where the coefficients $A_{n, j}$ are given by (4.9). 
Suppose that $n=1$. Applying Corollary 1.3 and the assumption that $A_{0, j} \in G_{p_{j}}$ to (4.9), we get

$$
\begin{aligned}
\left|A_{1, j}(z)\right| & \leq \sum_{l=j+1}^{k}\left(\begin{array}{c}
l \\
j+1
\end{array}\right)\left|A_{0, l}(z)\right|\left|\frac{f_{0,1}^{(l-j-1)}(z)}{f_{0,1}(z)}\right| \\
& \leq \sum_{l=j+1}^{k}\left(\frac{1}{1-|z|}\right)^{p_{l}+(l-j-1)(\max \{\beta, 1\}+1)+\varepsilon / 2} \leq\left(\frac{1}{1-|z|}\right)^{M_{1+j}+\varepsilon}
\end{aligned}
$$

for all $|z| \in[0,1) \backslash E$ and $j=0, \ldots, k-2$. This proves (4.10) for $n=1$.

To complete the proof it suffices to show that if, for a given $i \in\{2, \ldots, m-2\}$, the inequality

$$
M\left(r, A_{i-1, j}\right) \leq\left(\frac{1}{1-r}\right)^{M_{i-1+j}+\varepsilon / 3}
$$

holds for all $r \in[0,1) \backslash E$ and $j=0, \ldots, k-i$, then (4.10) is valid for $n=i$ and for $j=0, \ldots, k-i-1$.

Applying Lemma 3.2 to (4.7), we conclude that the functions $f_{q, j}, q=1, \ldots, n$, $j=1, \ldots, m-q$, are of the form $f_{q, j}=g_{q, j} / h_{q, j}$, where $g_{q, j}, h_{q, j} \in A(\mathbb{D})$ with $\max \left\{\sigma_{M}\left(g_{q, j}\right), \sigma_{M}\left(h_{q, j}\right)\right\} \leq \beta$. Therefore, by Corollary 4.2,

$$
\left|\frac{f_{i-1,1}^{(l-j-1)}(z)}{f_{i-1,1}(z)}\right| \leq\left(\frac{1}{1-|z|}\right)^{(l-j-1)(\max \{\beta, 1\}+1)+\varepsilon / 3}
$$

for all $|z| \in[0,1) \backslash E$ and $l=j+1, \ldots, k-i+1$. Applying (4.11) and (4.12) to (4.9), where $n=i$, results in

$$
\left|A_{i, j}(z)\right| \leq C \sum_{l=j+1}^{k-i+1}\left(\frac{1}{1-|z|}\right)^{M_{i-1+l}+(l-j-1)(\max \{\beta, 1\}+1)+2 \varepsilon / 3} \leq\left(\frac{1}{1-|z|}\right)^{M_{i+j}+\varepsilon}
$$

for all $|z| \in[0,1) \backslash E$ and $j=0, \ldots, k-i-1$. Hence (4.10) holds for $n=i$ and for all $j=0, \ldots, k-i-1$.

LEMMA 4.4. Under the assumptions of Lemma 4.3,

$$
A_{n, 0}(z)=A_{0, n}(z)+G_{n}(z)
$$

where

$$
G_{n}(z)=\sum_{j=2}^{n+1} \sum_{l=j}^{k-n+j-1}\left(\begin{array}{c}
l \\
j-1
\end{array}\right) A_{n-j+1, l}(z) \frac{f_{n-j+1,1}^{(l-j+1)}(z)}{f_{n-j+1,1}(z)}
$$


Moreover,

$$
M\left(r, G_{n}\right) \leq\left(\frac{1}{1-r}\right)^{M_{n+1}+\max \{\beta, 1\}+1+\varepsilon}
$$

for all $r \in[0,1) \backslash E$.

PROOF. Since (4.13) and (4.14) are valid by [7, Lemma 6.5], it follows that

$$
\left|G_{n}(z)\right| \leq \sum_{j=2}^{n+1} \sum_{l=j}^{k-n+j-1}\left(\begin{array}{c}
l \\
j-1
\end{array}\right)\left|A_{n-j+1, l}(z)\right|\left|\frac{f_{n-j+1,1}^{(l-j+1)}(z)}{f_{n-j+1,1}(z)}\right|
$$

The final assertion (4.15) follows by applying (4.10) to the coefficient functions $A_{n-j+1, l}$ and Corollary 4.2 to the logarithmic derivatives $f_{n-j+1,1}^{(l-j+1)} / f_{n-j+1,1}$, and by arguing similarly as in the proof of Lemma 4.3.

We return to the proof of Theorem 1.4(c). Let $n \geq 1$ be the smallest index such that (1.11) holds. Arguing by contradiction, we assume that equation (1.5) has $n+1$ linearly independent solutions $f_{0, q}$ such that

$$
\sigma_{M}\left(f_{0, q}\right)<\alpha=\max _{0 \leq j \leq k-1}\left\{\frac{p_{j}}{k-j}-1\right\}, \quad q=1, \ldots, n+1 .
$$

Note that (1.8) and (1.9) yield

$$
\sigma_{M}\left(f_{0, q}\right) \geq 1, \quad q=1, \ldots, n+1 .
$$

We aim for a contradiction, which, together with (1.7), shows that equation (1.5) has at least $k-n$ linearly independent solutions $f$ for which (1.12) is valid.

Let $\varepsilon>0$ be a small constant. Then Lemma 4.3, with $m=n+1$, and assumption (4.16) imply that there exists a solution $f_{n, 1} \not \equiv 0$ of (4.8) of the form $f_{n, 1}=g_{n, 1} / h_{n, 1}$, where $g_{n, 1}, h_{n, 1} \in A(\mathbb{D})$ with

$$
\max \left\{\sigma_{M}\left(g_{n, 1}\right), \sigma_{M}\left(h_{n, 1}\right)\right\}<\alpha .
$$

Moreover, taking (4.17) into account,

$$
M_{n+j} \leq \max _{n+j \leq l \leq k}\left\{p_{l}+(l-n-j)(\alpha+1)\right\} \leq(k-n-j)(\alpha+1)
$$

for $j=0, \ldots, k-q-1$, so that

$$
M\left(r, A_{n, j}\right) \leq\left(\frac{1}{1-r}\right)^{(k-n-j)(\alpha+1)+\varepsilon}
$$

for all $r \in[0,1) \backslash E$ and $j=0, \ldots, k-q-1$. Applying (4.16), (4.17) and (4.19) to $(4.15)$, it follows that

$$
M\left(r, G_{n}\right) \leq\left(\frac{1}{1-r}\right)^{(k-n)(\alpha+1)-2 \varepsilon}
$$


for all $r \in[0,1) \backslash E$. Let $\eta \in(\delta, 1)$, and let $F \subset[0,1)$ be the set in Lemma 4.1 (applied to $\left.A_{0, n}\right)$ with $\bar{D}(F) \geq \eta$. Then $\bar{D}(F \backslash E) \geq \eta-\delta>0$, and (4.21) and Lemma 4.1 yield

$$
M\left(r, A_{n, 0}\right) \geq M\left(r, A_{0, n}\right)-M\left(r, G_{n}\right) \geq\left(\frac{1}{1-r}\right)^{(k-n)(\alpha+1)-\varepsilon}
$$

for all $r \in F \backslash E$. Corollary 4.2 and inequality (4.18) show that

$$
\left|\frac{f_{n, 1}^{(j)}(z)}{f_{n, 1}(z)}\right| \leq\left(\frac{1}{1-|z|}\right)^{j(\alpha+1)-3 \varepsilon}
$$

for all $|z| \in F \backslash E$ and $j=1, \ldots, k-q$. By substituting $f=f_{n, 1}$ in (4.8), it follows that

$$
\left|A_{n, 0}(z)\right| \leq \sum_{j=1}^{k-n}\left|A_{n, j}(z)\right|\left|\frac{f_{n, 1}^{(j)}(z)}{f_{n, 1}(z)}\right| .
$$

This, with the aid of (4.20), (4.22) and (4.23), results in the contradictory inequality $(k-n)(\alpha+1)-\varepsilon \leq(k-n)(\alpha+1)-2 \varepsilon$.

4.3. Proof of (b). Suppose that (1.10) is valid. Then $\sigma_{M}(f) \leq \alpha$ for every solution $f$ of (1.5) by (1.7).

Conversely, suppose that all solutions $f$ of (1.5) satisfy $\sigma_{M}(f) \leq \alpha$, and assume that there is at least one coefficient $A_{j_{0}} \in G_{p_{j_{0}}}$ of (1.5) for which $p_{j_{0}} /\left(k-j_{0}\right)-1>\alpha$. Now, if $n \in\{0, \ldots, k-1\}$ is the smallest index such that $p_{n}$ satisfies (1.11), then, by Section 4.2, equation (1.5) has at least $k-n \geq 1$ linearly independent solutions $f$ such that

$$
\sigma_{M}(f)=\max _{0 \leq j \leq k-1}\left\{\frac{p_{j}}{k-j}-1\right\} \geq \frac{p_{j_{0}}}{k-j_{0}}-1>\alpha .
$$

This is a contradiction, and so (1.10) is valid.

\section{References}

[1] M. L. Cartwright, Integral Functions, Cambridge Tracts in Mathematics and Mathematical Physics, 44 (Cambridge University Press, Cambridge, 1956).

[2] Z.-X. Chen and K. H. Shon, 'The growth of solutions of differential equations with coefficients of small growth in the disc', J. Math. Anal. Appl. 297(1) (2004), 285-304.

[3] I. Chyzhykov, G. G. Gundersen and J. Heittokangas, 'Linear differential equations and logarithmic derivative estimates', Proc. London Math. Soc. 86(3) (2003), 735-754.

[4] I. Chyzhykov, J. Heittokangas and J. Rättyä, 'On the finiteness of $\varphi$-order of solutions of linear differential equations in the unit disc', J. Anal. Math. 209 (2009), 163-198.

[5] P. C. Fenton and J. Rossi, 'ODEs and Wiman-Valiron theory in the unit disc', J. Math. Anal. Appl. 367(1) (2010), 137-145.

[6] P. C. Fenton and M. M. Strumia, 'Wiman-Valiron theory in the disc', J. London Math. Soc. 79(2) (2009), 478-496.

[7] G. G. Gundersen, E. M. Steinbart and S. Wang, 'The possible orders of solutions of linear differential equations with polynomial coefficients', Trans. Amer. Math. Soc. 350(3) (1998), $1225-1247$. 
[8] J. Heittokangas, 'On complex differential equations in the unit disc', Ann. Acad. Sci. Fenn. Math. Diss. 122 (2000), 1-54.

[9] J. Heittokangas, 'Growth estimates for logarithmic derivatives of Blaschke products and of functions in the Nevanlinna class', Kodai Math. J. 30 (2007), 263-279.

[10] J. Heittokangas, R. Korhonen and J. Rättyä, 'Generalized logarithmic derivative estimates of Gol'dberg and Grinshtein type', Bull. London Math. Soc. 36 (2004), 105-114.

[11] J. Heittokangas, R. Korhonen and J. Rättyä, 'Linear differential equations with solutions in Dirichlet type subspace of the Hardy space', Nagoya Math. J. 187 (2007), 91-113.

[12] O. P. Juneja and G. P. Kapoor, Analytic Functions-Growth Aspects, Research Notes in Mathematics, 104 (Pitman, Boston, MA, 1985).

[13] R. Korhonen and J. Rättyä, 'Linear differential equations in the unit disc with analytic solutions of finite order', Proc. Amer. Math. Soc. 135(5) (2007), 1355-1363.

[14] R. Korhonen and J. Rättyä, 'Finite order solutions of linear differential equations in the unit disc', J. Math. Anal. Appl. 349 (2009), 43-54.

[15] I. Laine, Nevanlinna Theory and Complex Differential Equations (Walter de Gruyter, Berlin, 1993).

[16] B. Ja. Levin, Distribution of Zeros of Entire Functions, revised edition, Translations of Mathematical Monographs, 5 (American Mathematical Society, Providence, RI, 1980), translated by R. P. Boas et al.

[17] C. N. Linden, 'The minimum modulus of functions regular and of finite order in the unit circle', Q. J. Math. Oxford Ser. (2) 7 (1956), 196-216.

[18] J. Rättyä, 'Linear differential equations with solutions in Hardy spaces', Complex Var. Elliptic Equ. 52(9) (2007), 785-795.

I. CHYZHYKOV, Department of Mechanics and Mathematics, Lviv National University, Universytetska 1, Lviv 79000, Ukraine e-mail: ichyzh@lviv.farlep.net

J. HEITTOKANGAS, Mathematics, University of Joensuu, PO Box 111, 80101 Joensuu, Finland e-mail: janne.heittokangas@joensuu.fi

J. RÄTTYÄ, Mathematics, University of Joensuu, PO Box 111, 80101 Joensuu, Finland e-mail: jouni.rattya@joensuu.fi 